\title{
Cutaneous Vascular Neoplasm
}

National Cancer Institute

\section{Source}

National Cancer Institute. Cutaneous Vascular Neoplasm. NCI Thesaurus. Code C5354.

A benign, intermediate, or malignant vascular neoplasm that arises from the skin. 